\title{
Current Perspectives on Cardiomyopathies
}

\author{
Nandita Mehta and Sayyidah Aasima tu Nisa Qazi \\ Additional information is available at the end of the chapter
}

http://dx.doi.org/10.5772/intechopen.79529

\begin{abstract}
Cardiomyopathy is a disease of the heart muscle that can affect any age group or gender and can be acquired or inherited. Its literal meaning is "heart muscle disease" and refers to the deterioration of the function of the myocardium for any reason. Cardiomyopathy affects the shape, function, and/or electrical system of the heart. Patients with cardiomyopathy can present to anaesthesiologists in emergency situations or as elective cases. The patients are usually not aware of their condition and may be diagnosed at the time of pre-anaesthetic check-up. Advanced cases are always a challenge to the anaesthesiologist as they are most commonly complicated by progressive cardiac failure. Patients with cardiomyopathy are often at risk of dysrhythmias or sudden cardiac death. This chapter describes the main features and perioperative management of patients with cardiomyopathies undergoing non-cardiac surgery. In general, the optimal anaesthetic management of patients with cardiomyopathy requires good preoperative assessment, close perioperative monitoring, suitable anaesthetic agents, optimal perioperative fluid management, and an overall stable hemodynamic status.
\end{abstract}

Keywords: anaesthesiologist, cardiomyopathy, anaesthetic management

\section{Introduction}

The first officially accepted definition of cardiomyopathy was given by WHO in 1980 which defined it as "heart muscle diseases of unknown cause" to differentiate cardiomyopathy from cardiac dysfunction which occurs due to known cardiovascular diseases such as hypertension, coronary artery disease, or valvular disease [1]. WHO reclassified cardiomyopathies in 1995 to include diseases of myocardium associated with cardiac dysfunction that were earlier excluded. They expanded this criterion in order to include all known causes of cardiomyopathy and is based on anatomical and physiological features. It includes three main types of cardiomyopathy: hypertrophic (HCM), dilated (DCM), and restrictive (RCM). 
In 2006, American Heart Association (AHA) in their document entitled "Contemporary Definition and Classification of the Cardiomyopathies" defined cardiomyopathies as "a heterogeneous group of diseases of the myocardium associated with mechanical and/or electrical dysfunction that usually (but not invariably) exhibit inappropriate ventricular hypertrophy or dilation and are due to a variety of causes that frequently are genetic. Cardiomyopathies either are confined to the heart or are a part of generalised systemic disorders, often leading to cardiovascular death or progressive heart failure-related disability." According to the new AHA classification, cardiomyopathies are divided into two broad groups: primary cardiomyopathies and secondary cardiomyopathies. Primary cardiomyopathies encompass those that are exclusively or predominantly confined to the heart muscle and are acquired, genetic, or of mixed origin. Secondary cardiomyopathies include the subset of multiorgan involving diseases, which cause involvement of the heart as a part of their pathophysiology. In spite of this detailed classification, some confusion may arise because some primary cardiomyopathies may have associated extra cardiac components while as a few secondary cardiomyopathies can affect the heart exclusively.

\section{Classification of primary cardiomyopathies:}

\begin{tabular}{ll}
\hline Genetic & Hypertrophic cardiomyopathy \\
& Arrhythmogenic right ventricular cardiomyopathy \\
& Left ventricular noncompaction \\
& Glycogen storage disease \\
& Conduction system disease (Lenègre's disease) \\
& Ion channelopathies: long QT syndrome, Brugada syndrome, short QT syndrome \\
Mixed & Dilated cardiomyopathy \\
& Primary restrictive nonhypertrophied cardiomyopathy \\
Acquired & Myocarditis (inflammatory cardiomyopathy): viral, bacterial, rickettsial, fungal, parasitic (Chagas \\
& disease) \\
& Stress cardiomyopathy \\
& Peripartum cardiomyopathy
\end{tabular}

\section{Classification of secondary cardiomyopathies:}

\begin{tabular}{ll}
\hline Infiltrative & $\begin{array}{l}\text { Amyloidosis } \\
\text { Gaucher's disease } \\
\text { Hunter's syndrome }\end{array}$ \\
Storage & Hemochromatosis \\
& Glycogen storage disease \\
& Niemann-Pick disease
\end{tabular}




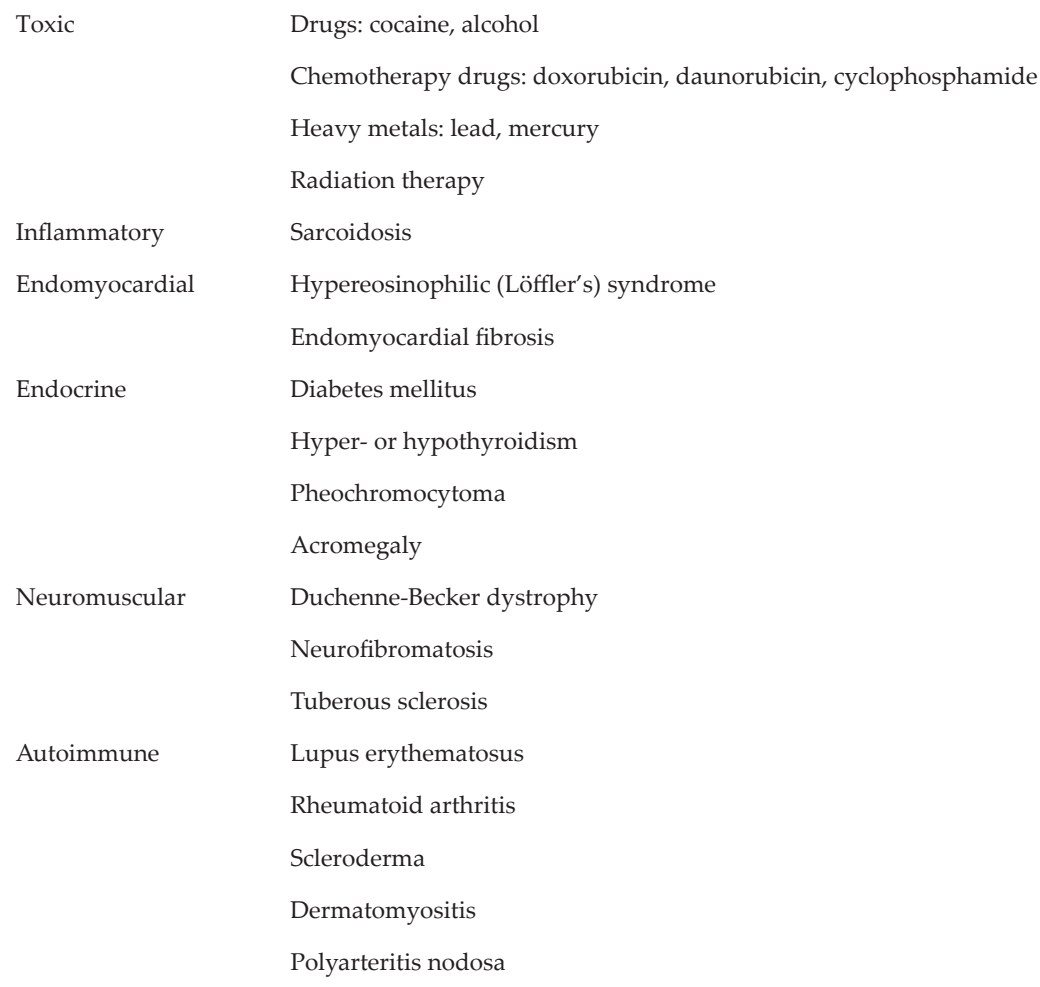

European Society of Cardiology in 2008 introduced a classification in which they accommodated five specific types of cardiomyopathies along with their genetic involvement: dilated, hypertrophic, arrhythmogenic, restrictive, and unclassified [2]. They further divided them into familial (genetic) or non-familial (non-genetic).

The most recent classification known as the MOGE(S) classification system had been introduced which is based on phenotype and genotype an it incorporates information on structural and functional abnormalities $(\mathrm{M})$, organ involvement $(\mathrm{O})$, genetics $(\mathrm{G})$, aetiology $(\mathrm{E})$, and disease severity (S) associated with the condition [3]. However, it cannot be considered as complete as it does not include certain cardiomyopathies like postpartum cardiomyopathy or the risk of sudden death and is very complex to use. The MOGES classification is beyond the scope of this review so we do not discuss it here.

\section{Pathophysiology}

Cardiomyopathy itself can present as either systolic dysfunction or diastolic dysfunction, which in turn are both related to the ventricular dysfunction. 
Systolic dysfunction: This type of dysfunction is mainly seen in dilated cardiomyopathy. The predominant pathophysiology is a global decrease in myocardial contractility, which in turn leads to reduction in left ventricular ejection fraction. In the initial phases, the heart tries to compensate this change by increasing the size of left ventricular cavity which allows for an improvement in stroke volume with an associated improved force of contraction. As the disease progresses, these compensatory mechanisms prove to be inadequate in maintaining the cardiac output, eventually leading to the failure of left heart.

Diastolic dysfunction: This is the most common type of dysfunction associated with the cardiomyopathies, occurring in HCM, RCM, and other types of cardiomyopathies. The main pathophysiology is impairment of filling of blood in the left ventricle, which leads to increase in the left ventricular filling pressures. During the beginning of the diastolic phase of a normal cardiac cycle, left ventricle undergoes the phase of Isometric relaxation (which is an energy dependent process) just before the start of left ventricle filling phase. This relaxation continues in the early left ventricular filling phase. The later part of the left ventricular filling is a passive process and depends on the compliance of the left ventricle. Diastolic dysfunction can be because of the impairment of any of the two phases: active relaxation or left ventricular compliance or a combination of the two. Ischaemia mainly affects the phase of isometric relaxation, while intrinsic myocardial pathologies including fibrosis or external restriction due to pericardial diseases may lead to a reduction in left ventricular compliance.

The main types of cardiomyopathy that we come across clinically in our day-to-day practice are:

- Dilated cardiomyopathy

- Hypertrophic cardiomyopathy

- Restrictive cardiomyopathy

- Arrhythmogenic right ventricular dysplasia

- Peripartum cardiomyopathy

Some other types of cardiomyopathy are known as "unclassified cardiomyopathy." Another type of cardiomyopathy known as Takotsubo cardiomyopathy has been recently listed and is also known as "stress-induced cardiomyopathy," or broken heart syndrome.

Therefore, we focus our attention towards the commonest types of cardiomyopathies in this chapter.

\section{Dilated cardiomyopathy}

Dilated cardiomyopathy (DCM) is defined by cardiac enlargement with impaired systolic function of one or both ventricles. It is defined by the presence of: 
a. Fractional myocardial shortening $<25 \%$ and/or ejection fraction $<45 \%$.

b. Left ventricular end-diastolic diameter $>117 \%$ excluding any known cause of myocardial disease.

Familial DCM contribute about $20-48 \%$ of all DCM and can defined by

- the presence of two or more affected relatives with DCM meeting the above criteria or

- a relative of a DCM patient with unexplained sudden death before the age of 35 .

The prevalence of DCM is 920/100,000 individuals and is common in Afro-Caribbean population. It is the commonest form of cardiomyopathy and is the third most common cause of congestive heart failure. DCM is the commonest indication for heart transplantation. In $30-40 \%$ patients, it is transmitted in an autosomal dominant fashion while in others it can be post viral or idiopathic. It can be of ischemic or nonischemic variety with ischemic type being related to atherosclerosis or CAD. The nonischemic variety may present itself secondary to the use of chemotherapeutic agents (doxorubicin and adriamycin), infections (Coxsackie virus, HIV, cytomegalovirus Chagas' disease, trichinosis, toxoplasmosis, Lyme disease, and leptospirosis), drug abuse (alcohol, heroin, cocaine, and methamphetamines), or as peripartum cardiomyopathy.

\subsection{Pathophysiology}

Dilated cardiomyopathy presents with a decrease in LV ejection fraction (LVEF) as described earlier, congestive heart failure (CHF) or as ventricular arrhythmias. Initially, the ventricle dilates to increase the force of contraction and stroke volume in order to maintain the cardiac output (Frank-Starling law); however, as the disease progresses, these compensatory mechanisms gradually fail, leading to the ventricular failure and ultimately failure to maintain the cardiac output $(\mathrm{CO})$.

\subsection{Signs and symptoms}

The patients of dilated cardiomyopathy present with symptoms like dyspnea, orthopnea, fatigue, weakness, and oedema in the lower extremities. Physical findings are similar to those seen in CHF. Some patients complain of dyspnea on exertion that may look like angina pectoris. Patients may have jugular venous distention, crepitation on auscultation, resting tachycardia, audible s3 and s4 heart sounds, pulmonary oedema, and cardiomegaly. Mitral and/or tricuspid regurgitation may be audible clinically if the ventricular dilation is marked.

The ECG may show ST-T segment abnormalities, atrial fibrillation, intraventricular conduction defects, and PVCs. The echocardiography reveals dilated cardiac chambers, global hypokinesia, low EF/fractional shortening, raised LVEDP, mitral or tricuspid regurgitation and/or mural thrombi. Right-sided cardiac catheterisation using a Swan Ganz Catheter reveals a high 
pulmonary capillary wedge pressure, high systemic vascular resistance, and a low cardiac output. Additional laboratory tests carried out may reveal raised brain natriuretic peptide levels.

\subsection{Treatment}

Management of DCM begins with lifestyle modifications such as adequate rest, weight control, low sodium diet, fluid restriction, stopping alcohol intake and smoking, and less physical activity during periods of cardiac decompensation.

Medical management: The patients of DCM are at increased risk of pulmonary and systemic thromboembolisation due to stasis of blood in the dilated hypokinetic cardiac chambers. Anticoagulation therapy with warfarin or dabigatran is often indicated in these patients. The risk of embolisation is the highest in patients with atrial fibrillation, severe left ventricular dysfunction, a previous history of thromboembolism, or echocardiographic evidence of a mural thrombus. Other medications like angiotensin-converting enzyme inhibitors and angiotensin antagonists, diuretics, beta blockers, vasodilators, digoxin, antiarrhythmics, and statins can be prescribed to keep the condition under control.

Patients with a LVEF $<30 \%$ and an intraventricular conduction defect with wide QRS complex $\geq 130$ ms may lack synchronised contraction of both ventricles. Resynchronisation of right and left ventricle with biventricular pacing using a cardiac resynchronisation therapy device (CRT-D) can restore synchronous contraction of both ventricles, shorten the QRS interval, decrease left ventricular size and improve systolic function, stroke volume and the overall survival rate of patients.

Heart transplantation is the definitive treatment and the most common indication for transplantation in patients with DCM for both adults and children. Patients that are likely to benefit highly from a heart transplant include patients who were previously very active, $<60$ years of age who show intractable symptoms of congestive heart failure despite optimal medical therapy.

\subsection{Prognosis}

Symptomatic patients with DCM who are referred to tertiary medical centres for care have a high 5-year mortality rate (50\%). If the cardiomyopathy involves both the right and left ventricles, the prognosis is very poor. Haemodynamic abnormalities that predict a poor prognosis include:

- an ejection fraction $<25 \%$,

- pulmonary capillary wedge pressure $>20 \mathrm{~mm} \mathrm{Hg}$,

- cardiac index $<2.5 \mathrm{~L} / \mathrm{min} / \mathrm{m}^{2}$,

- pulmonary hypertension,

- systemic hypotension, and

- an increased central venous pressure. 


\subsection{Anaesthetic management for non-cardiac surgery}

Any major surgeries on these patients can be associated with morbidity and mortality, therefore, requires planning. Optimisation of congestive heart failure (CHF) at least for a week before the planned surgery is advisable. In critically ill or patients undergoing a high-risk procedure or those in which CHF is not appropriately managed, intra-arterial BP line should be inserted preoperatively. Premedication should be tailored according to the patient's requirement and may include short acting anxiolytic and/or sedative. Regional anaesthesia or nerve blocks alone or in combination with general anaesthesia can help us achieve the set goals of anaesthesia with a minimal haemodynamic compromise. However, the ongoing anticoagulation therapy may limit the option of regional anaesthesia. American Society of Regional Anaesthesia (ASRA) guidelines must be strictly followed if the patient is on an anticoagulation therapy.

The goals of anaesthesia are to [4]:

- minimise any negative inotropic effect of anaesthetic drugs.

- prevent increases in afterload.

- maintain preload despite increased left ventricular end-diastolic pressure.

- maintain perfusion and control arrhythmias,

- avoid hypotension and tachycardia.

- avoid overdose of medications during induction as the circulation time of drugs is slow.

These patients can become haemodynamically unstable due to the depressant effect of anaesthetic agents, fluid shifts and ongoing blood loss, which add to the already poor myocardial function due the cardiomyopathy. Propofol, thiopentone and inhalational agents cause vasodilation and myocardial depression. Benzodiazepines like midazolam and nitrous oxide may cause cardiovascular depression. Etomidate, ketamine, and narcotics like opioids are the ones that have minimal adverse haemodynamic response. We need to use a balanced anaesthetic technique. Slow induction should be carried out. Response of induction agents may be delayed due to prolonged circulation time, so slow and titrated doses of anaesthetic agents should be administered. Additional doses may not be required.

Optimal pain management helps to maintain haemodynamic stability. Regional anaesthesia may be a source of excellent postoperative pain relief, reducing the episodes of sympathetically mediated tachycardia, and afterload increases.

Monitoring: In addition to basic monitoring, central venous pressure (CVP) monitoring allows us to measure preload and central venous saturation $\left(\mathrm{ScVO}_{2}\right)$. It provides for an access to administer inotropes and vasoconstrictors if needed. Direct intra-arterial pressure monitoring enables early identification of haemodynamic alterations by beat-to-beat measurement of BP. Pulmonary artery pressure monitoring is useful in patients undergoing high-risk or emergency surgery or those in whom large fluid shifts are anticipated. The role of noninvasive methods to estimate cardiac output as well as estimate global end-diastolic volumes, the extravascular lung water, and other indices if available are invaluable for assessing cardiac function. 
Transesophageal echocardiography (TEE) is also helpful as it identifies causes of hypotension, response to fluid therapy or inotrope support, estimates preload, cardiac output, diastolic dysfunction, valve function, and regional wall motion abnormalities.

\section{Hypertrophic cardiomyopathy}

Hypertrophic cardiomyopathy can be defined as abnormal LV thickening without chamber dilation that is usually asymmetrical, develops in the absence of an identifiable cause (e.g. aortic valvular stenosis[AS] and hypertension), and is associated with myocardial fibre disarray.

Hypertrophic cardiomyopathy (HCM) is very common and can affect people of any age group. It affects both sexes equally. It is a cause of sudden cardiac arrest and death in apparently healthy young people, including young athletes. HCM is a relatively common inherited disorder with an autosomal dominant pattern of inheritance with variable expression and has a prevalence of 1 in 500 .

Defects of at least 11 genes and $>1440$ mutations sites demonstrate its genomic heterogeneity.

\subsection{Pathophysiology}

The underlying structural abnormalities in HCM are:

1. myocardial cell disarray where the cells are rearranged in a disorganised pattern as opposed to a normal parallel myocyte arrangement;

2. coronary microvasculature dysfunction due to increased wall to lumen ratio; and.

3. remodelling changes occurring in the heart.

These changes in HCM patients lead to diastolic dysfunction, impaired coronary reserve, supraventricular and ventricular dysrhythmias, and sudden cardiac arrest. Left ventricular remodelling can include fibrosis, focal, diffuse, asymmetric, or concentric hypertrophy, as well as decrease in the cavity size. The most common subtype of HCM presents as hypertrophy of the septum and the anterio-lateral free wall.

LV outflow tract obstruction (LVOTO) occurs in HCM, and initially it was proposed that the basal septal hypertrophy encroaching on the LVOT caused the obstruction of the tract. However, more recent studies have pointed towards the fact that during ventricular systole, flow against an abnormally positioned mitral valvular apparatus results in a Venturi effect on the anterior leaflet of the mitral valve and induces systolic anterior movement (SAM) of the anterior mitral valve leaflet. The mitral valve apparatus abnormalities can include hypertrophied papillary muscles touching the septum, elongated mitral leaflets, anterior displacement of papillary muscles, or anomalous insertion of the papillary muscle onto the anterior mitral leaflet. LVOTO can be precipitated or aggravated by increased contractility of the heart or tachycardia or decreased end-diastolic volume or systemic vascular (arterial) resistance. With HCM, incidence of diastolic dysfunction is more than LVOTO. 
Myocardial ischaemia is present in patients with $\mathrm{HCM}$, irrespective of the presence or absence of coronary artery disease. Myocardial ischaemia is precipitated by several factors including

- a mismatch between ventricular mass and coronary artery size,

- increased oxygen consumption due to hypertrophy,

- abnormal coronary arteries,

- decreased diastolic filling time,

- increased LVEDP compromising coronary perfusion, and

- the presence of a metabolic derangement regarding the utilisation of oxygen at the cellular level.

Supraventricular or ventricular dysrhythmias are relatively common in these patients due to the presence of disorganised cellular architecture, expanded interstitial matrix and myocardial scarring. They are the cause of incidence of sudden arrest in this group of cardiomyopathy.

\subsection{Signs and symptoms}

The clinical presentation of HCM varies widely. These patients can present early in their life with debilitating symptoms or can live for decades asymptomatically while some others die suddenly. The most frequent symptoms include dyspnea, dizziness, exercise intolerance, angina, syncope, and/or sudden death.

Physical examination may be normal at rest but may reveal a double apical impulse, gallop rhythm, a systolic murmur and thrill in the presence of functional LVOTO. It is rare, but some people with hypertrophic cardiomyopathy can suffer sudden cardiac arrest during a vigorous physical workout. The physical activity can trigger dangerous arrhythmias leading to sudden death.

The major risk factors for sudden cardiac death are:

- a family history of sudden death;

- unexplained syncope;

- extreme hypertrophy of the left ventricular wall $(0.30 \mathrm{~mm})$;

- non-sustained ventricular tachycardia (VT)

Electrocardiography (ECG) changes include left atrial (LA) enlargement, pathologic Q waves, high QRS voltage complexes, ST segment depression, and inverted T waves in at least two or more consecutive leads.

Echocardiography can easily demonstrate the presence of myocardial hypertrophy. Ejection fraction is usually $>80 \%$, reflecting the increase in force of contraction of the heart. Echocardiography can also assess the mitral valvular apparatus, the presence of mitral regurgitation, and the 
presence of LVOTO by demonstrating turbulent flow across the aortic valve. Pressure gradients across the LVOT can be measured. Echocardiography is useful in evaluating diastolic function of the heart.

Invasive measures like cardiac catheterisation allow direct measurement of the increased left ventricular end-diastolic pressure and the pressure gradient between the left ventricle and the aorta.

The definitive diagnosis of HCM can be made by an endomyocardial biopsy and DNA analysis, but these diagnostic modalities are usually reserved for patients in whom the diagnosis cannot be established by non-invasive means.

\subsection{Treatment}

The varied clinical profile of HCM makes it difficult to establish some precise guidelines for the treatment of this condition. The treatment plan should be titrated according to individual patient requirements. However, it is prudent to mention that the patients who are at high risk for sudden death should receive aggressive treatment.

Medical management: Pharmacotherapy is aimed at reducing LVOTO, improving diastolic filling, and possibly decreasing myocardial ischaemia. A variety of medical therapies have been used in these patients with the aim of altering the natural history. These include $\beta$-blockers, $\mathrm{Ca}^{2+}$ channel antagonists (verapamil or diltiazem), and disopyramide regimens, all of which seem to be effective as compared to no treatment in HCM patients. Most of these drugs used help HCM patients to improve their symptoms by reducing or eliminating the LVOT pressure gradient. These medications also reduce LVOTO during exercise by blunting the sympathetic response and are thus useful in treating the symptoms and attenuating the risk of sudden cardiac arrest. Atrial fibrillation often develops in these patients. It is associated with an increased risk of thromboembolism and congestive heart failure. Amiodarone is the most effective drug for prevention of repeated episodes of atrial fibrillation in these patients. Long-term anticoagulation is indicated in the patients with recurrent or chronic atrial fibrillation to prevent thromboembolic episode reducing the mortality and morbidity associated with it.

More recently, perhexiline, which augments myocyte energy supply, has been shown to improve diastolic dysfunction and symptomatology, but detailed studies are yet to follow.

Alcohol septal ablation [5]: Ethanol can be infused into the septal branches of the left anterior descending coronary artery and induce a targeted septal myocardial infarction (MI). Alcohol septal ablation is associated with some serious hazards, most common being the right bundle branch block, which has a post-procedural incidence of approximately $50 \%$. Other complications include remote MI due to collateral circulation or an ethanol injection into the incorrect coronary, coronary dissection, ventricular septal rupture, heart failure, and heart block.

Surgical management: The American and European Colleges of Cardiology recommend myectomy in patients with: 
1. labile obstruction and peak LVOT pressure gradients $\geq 50 \mathrm{~mm} \mathrm{Hg}$ during exercise or provocation and resting gradients $>30 \mathrm{~mm} \mathrm{Hg}$ and.

2. NYHA class II through IV symptoms refractory to medical therapy.

Some patients may be candidates for implantable cardioverter-defibrillator (ICD) implantation while as principal surgical option is surgical myomectomy.

Prognosis: The overall mortality rate of HCM is $1 \%$ per annum. However, some patients at higher risk of sudden death as described before have an annual mortality rate of $5 \%$.

Patients undergoing non-cardiac surgery: Most of the time, the patients with HCM are asymptomatic when they show up in the PAC clinic for elective surgeries. In the absence of signs and symptoms, the ECG findings may suggest that the patient has underlying HCM.

Initial patient evaluation should be aimed at determining the disease severity by assessing functional status of the patient, personal and family cardiac history, the presence or absence of cardiac and respiratory symptoms, history of rhythm disturbances, current medications, and previous strokes, or congestive heart failure history. During physical examination, all murmurs should be evaluated for dynamic changes with rest and exertion, and patients with murmurs that do not fulfil the criteria of a benign murmur should undergo an echocardiographic examination before surgery. Patients should be instructed to continue their rate controlling medications and maintain proper hydration preoperatively. Moreover, the presence of an automatic ICD and if it has been recently checked should be determined.

Preoperative management: A lot of patients with HCM may experience perioperative cardiac events like MI, congestive heart failure, severe hypotension, and supraventricular and ventricular tachydysrhythmias. Therefore, we need to focus on understanding the basic pathophysiology of the events and adjust our anaesthetic plans according to the patient needs.

In patients with $\mathrm{HCM}$, preoperative administration of anti-anxiety medications may help to reduce anxiety and prevent the activation of anxiety-induced sympathetic response. Adequate preoperative intravenous fluid administration may help in preventing LVOTO and minimise the effect of positive pressure ventilation on central blood volume.

For patients who have an ICD in situ, the device should be turned off just before the surgery and an external defibrillator should be readily available and the ICD should be positively reactivated in the recovery room.

The anaesthetic goals are [4]:

- maintenance of sinus rhythm;

- reduction in sympathetic activity to reduce chronotropy and inotropy;

- the maintenance of systemic vascular resistance;

- maintenance of left ventricular filling. 
Tachycardia, arrythmias, and decreases in afterload will exacerbate LVOTO and may cause haemodynamic deterioration. In addition to this, increases in contractility (chronotropy) and decreases in preload will accentuate LVOTO. Therefore, the principle of treatment for hypotension is volume expansion (including increasing preload in the Trendelenburg position) and use of drugs that increase systemic vascular resistance without a positive inotropic or chronotropic response (e.g. phenylephrine and vasopressin). Sympathetic response secondary to patient anxiety, intubation process, and surgical site incision and acute changes in preload, afterload, and contractility secondary to the pharmacological effects of anaesthetic agents, blood loss during surgery, and postoperative pain can precipitate haemodynamic collapse. DC cardioversion may be necessary in case of sudden onset of atrial fibrillation that is haemodynamically unstable.

Although both general and neuraxial anaesthesia can be used, it is important to have a clear understanding of the haemodynamic changes associated with each option. Depending on the route of the anaesthetic drugs chosen, close monitoring and titration of the medications affecting heart rate, preload, afterload, contractility of myocardium, and sympathetic activity are important. Neuraxial techniques may also be considered. In general, a slow controlled titration of medication via an epidural is preferred over a single dose spinal anaesthesia with the aim of maintaining preload and afterload and avoiding sympathetic stimulation. Regional anaesthesia can be an invaluable tool to manage postoperative pain and in turn prevent the activation of sympathetic response in these patients.

In addition to the standard American Society of Anaesthesiologists monitoring requirements, an intra-arterial catheter and/or non-invasive pulse plethysmographic variability (PPV) index monitor and central venous pressure (CVP) monitoring may be considered. The overall haemodynamic goals include maintaining the mean arterial blood pressure at $>65-70 \mathrm{~mm} \mathrm{Hg}$ to maintain coronary perfusion pressure to the subendocardium in the hypertrophied heart. The most useful monitoring tool for patients undergoing high-risk surgery is TEE. TEE can determine whether haemodynamic alterations are caused by hypovolemia, increased LVOTO or SAM, or LV systolic dysfunction.

Postoperative management: Patient with HCM should be continuously monitored in the postoperative room. All factors that activate a sympathetic response like pain, hypothermia, shivering, anxiety, hypoxia, and hypercarbia should be immediately addressed. The maintenance of euvolemia and prompt treatment of hypotension is very important.

\section{Restrictive cardiomyopathy}

Restrictive cardiomyopathy (RCM) is a disorder of the myocardium that occurs due to increased myocardial stiffness (decreased compliance) that leads to impaired ventricular filling. Size of both ventricle chambers and systolic function usually remains normal or near-normal until later stages of the disease. RCM may arise as a result of either inherited or acquired predispositions and diseases or a combination of the both, and can broadly be classified as infiltrative, non-infiltrative, storage disease, and endomyocardial fibrosis. Restrictive 
cardiomyopathy is prevalent in tropical regions of the world, where incidence of endomyocardial fibrosis is high. In non-tropical regions, idiopathic fibrosis is the common cause and is associated with increasing age. Other rare causes of RCM include amyloidosis, haemochromatosis, sarcoidosis, and eosinophilic endocarditis.

\subsection{Pathophysiology}

RCM is characterised by contracted stiff ventricles with progressive impairment of diastolic filling, leading to the haemodynamic problem of a low preload but high ventricular filling pressure. This pattern of diastolic dysfunction leads to dilation of the atria and elevation of mean atrial pressures, resulting in biventricular "backward heart failure" manifesting itself as pulmonary venous congestion leading to dyspnea as well as systemic venous pressure elevation resulting in peripheral oedema. Systolic function is preserved in most cases. However, in spite of intact systolic function, the restrictive pathology on true ventricular preload limit the stroke volume, resulting in low cardiac output and ultimately hypoperfusion of the tissues.

\subsection{Signs and symptoms}

RCM presents with signs and symptoms of both right and left heart failure. Patients complain of exercise intolerance because of diminished cardiac output. Patients often have a low volume pulse, an audible third heart sound, regurgitant murmurs, and a raised JVP with rapid X and $Y$ descent that increases or fails to decrease on inspiration. Low blood pressures are often seen, complicating heart failure management. Pulmonary oedema is uncommon. Syncope occurs occasionally, often exertional, reflecting the limited ability of the heart to increase diastolic filling and is an ominous sign. Syncope may also be aggravated by antihypertensive medications. Concomitant autonomic neuropathy can precipitate orthostatic hypotension as can volume contraction from nephrotic syndrome.

Arrhythmias and conduction disturbances are frequent. Less frequent cardiac manifestations include dynamic LV outflow obstruction, often confused with hypertrophic cardiomyopathy; cardiac ischaemia caused by amyloid deposition in intramural coronary arteries; and intracardiac thrombosis caused by atrial wall standstill, with a risk for systemic embolisation.

The ECG may demonstrate conduction abnormalities. The chest X-ray shows signs of pulmonary congestion and/or pleural effusion, but cardiomegaly is absent. Echocardiographybased two-dimensional and Doppler are essential for determining diastolic dysfunction and for distinguishing patients with RCM from patients with restrictive physiology because of constrictive pericarditis. Echocardiography may also provide information to suggest a specific diagnosis such as the presence of regional wall motion abnormalities in a non-coronary distribution and aneurysms, which would raise the suspicion for cardiac sarcoidosis (CS). Cardiac magnetic resonance (CMR) imaging can aid in the diagnostic process, but the use should be determined on an individual basis. Endomyocardial biopsy (EMB) may be helpful for establishing a diagnosis in some cases. Ultimately, diagnosis of any of the RCMs relies on a constellation of clinical, laboratory, and imaging findings. 


\subsection{Management}

Medical management: Treatment of RCM includes treating the underlying cause (if identified) and heart failure management. Diuretics are the mainstay of treatment to reduce volume overload. However, volume status in patients with RCM may be challenging to manage, as patients with RCM rely on high filling pressures to maintain cardiac output and excessive diuresis may result in tissue hypoperfusion. Digoxin must be used with great caution because it is potentially dysrhythmogenic in patients with amyloidosis. The use of $\beta$-blockers or calcium channel blockers to increase filling time or to manage arrhythmias should be carefully introduced, as some patients may be intolerant. Angiotensin-converting enzyme inhibitors and angiotensin II receptor blockers may also be considered, but the proof of benefit is lacking, and these agents may not be well tolerated. Anticoagulation is required in patients with atrial fibrillation, mural thrombus, or evidence for systemic embolisation and may be helpful in most patients because of propensity for thrombus formation in the left atrial appendage.

Surgical management: No corrective surgery has yet been proposed that would be $100 \%$ effective in improving the heart function in RCM. Advanced heart failure therapies, including cardiac transplantation, may be beneficial for selected patients. Heart transplantation is the only effective surgery that can be offered to the patients with restrictive cardiomyopathy. It may be the best option for those who are already symptomatic at the time of diagnosis or in whom reactive pulmonary hypertension exists. Left ventricular assist device (LVAD) therapy may be particularly applicable in patients with RCM as a bridge to transplant or as definitive therapy.

\subsection{Patients posted for non-cardiac surgery}

RCM presents a huge challenge for anaesthetists due to the high risk of morbidity and mortality. General anaesthesia causes vasodilation, suppresses the myocardium, and reduces venous return. The latter can be worsened by intermittent positive pressure ventilation resulting in cardiac arrest. Invasive arterial blood pressure monitoring and transesophageal echocardiography are useful in identifying the causes of cardiovascular instability [6].

The overall aims of anaesthesia are:

- sinus rhythm to be maintained if possible;

- to maintain adequate filling pressures;

- to maintain SVR in the presence of relatively fixed cardiac output.

- to manage electrolyte disturbances;

- to use anaesthetic agents with minimal cardiovascular effect like ketamine or etomidate.

\section{Arrhythmogenic right ventricle cardiomyopathy}

Arrhythmogenic right ventricle cardiomyopathy (ARVC) is characterised by structural abnormalities and cardiac dysfunction of mainly the right ventricle, but it can also involve the left 
ventricle. It is a rare type of cardiomyopathy. It occurs if the muscle tissue in the right ventricle dies and is replaced by a scar tissue. This disrupts the pathway of the heart's electrical signals leading to arrhythmias.

ARVC usually affects teenagers or young adults. ARVC has a prevalence of 1 in 5000 among healthy young people. ARVC is seen in up to $20 \%$ of all causes of sudden death in young people. It is a complex genetic condition due to its genetic variation.

\subsection{Pathophysiology}

Histologically, the myocardial cells are replaced by the adipose and fibrous tissues. These alterations can form a re-entry electrical circuit triggering arrhythmias. ARVC usually starts as a localised disease with regional wall abnormalities. As the disease progresses, the right ventricle continues to lose the healthy tissue and dilates and becomes thin walled. Patients can develop right bundle branch block before they finally present with the symptoms of right ventricular failure between the fourth and fifth decades of life.

\subsection{Signs and symptoms}

Young patients often present with syncope, arrhythmia, cardiac arrest, or sudden death. The diagnosis of ARVC should be considered in:

- Young male athletes with cardiac symptoms.

- ECG showing ventricular tachycardia with left bundle branch block morphology.

- T-wave inversion in leads V1-V3.

- Premature ventricular complexes with left bundle branch block morphology.

- Spontaneous non-sustained ventricular tachycardia.

An accurate diagnosis of ARVC is important due to the high risk of drug-resistant arrhythmias and sudden cardiac death. The diagnosis can be established by myocardial biopsy, which shows adipocytes and fibrous tissue. However, these changes can be localised and may not be present at the exact site of biopsy. The availability of cardiac MRI and the gadolinium enhancement techniques are now fundamental in diagnosing ARVC eliminating the need for biopsies.

\subsection{Management}

The main aim of medical management is to prevent or reduce the risk of fatal arrhythmias.

- Sotalol, verapamil, and amiodarone can be used.

- Due to the recurrence of arrhythmias and drug resistance, continuous Holter monitoring, or an electrophysiological study may be required. Catheter ablation can be used as a palliative rather than a curative intervention. It is indicated in patients with monomorphic VT due to alocalised ARVC, with a drug-resistant arrhythmia, or with frequent intervention following ICD implantation [7]. 
- Early placement of an ICD may be lifesaving.

- In some exceptional cases, heart transplantation may be required.

\section{Peripartum cardiomyopathy}

Peripartum cardiomyopathy is a rare, dilated form of cardiomyopathy of unknown cause that occurs during the peripartum period, that is, the third trimester of pregnancy until 5 months after delivery. Peripartum cardiomyopathy (PPCM) is a major concern for anaesthetists and can occur in 1 in 10,000 pregnancies, but it is higher in subsequent pregnancies [8]. Patients may present with severe heart failure during the third trimester or up to 5 months postpartum. Many of these patients deliver via a normal vaginal delivery without complications; however, a few may require a Caesarean section.

Risk factors include maternal age $>30$ years, multiparty, African descent, obesity, multiple pregnancy, hypertensive disorders, tocolytic therapy, viral infection, and cocaine use.

Diagnostic criteria of peripartum cardiomyopathy: The diagnosis of PPCM is usually made after the other causes of acute heart failure have been excluded. The criteria are:

- Heart failure developing towards the end of pregnancy or up to 5 months' postpartum

- Absence of another identifiable cause of cardiac failure

- Absence of cardiac symptoms or disease before late pregnancy

- Left ventricular dysfunction - defined as an $\mathrm{EF}<45 \%$ or reduced fractional shortening of $<30 \%$

\subsection{Signs and symptoms}

The patients usually present with sign and symptoms of heart failure: dyspnea, fatigue, and peripheral oedema. In early stages, these signs may mimic the presenting features of normal late pregnancy.

Echocardiography may show new onset of unexplained LV dysfunction and documentation of a new finding of dilated cardiac chambers with LV systolic dysfunction during the period surrounding parturition.

\subsection{Treatment}

The main aim of treatment is to relieve the symptoms of heart failure. Diuretics, vasodilators, and digoxin can be used effectively. During pregnancy, vasodilation is accomplished with hydralazine and nitrates. Intravenous immunoglobulin may have a beneficial effect. Thromboembolic complications are not uncommon, and anticoagulation may be required in most patients. Heart transplantation may be considered in patients who do not improve over time. 


\subsection{Prognosis}

The mortality in this group of patients is as high as 30-60\% due to pulmonary oedema and systemic embolisation with most deaths occurring mostly within 3 months of delivery.

\subsection{Anaesthetic management}

We have a very little literature regarding the anaesthetic management of PPCM yet. Optimum fluid management and avoiding myocardial depression are the major concerns for anaesthetists.

According to a few case reports, both general anaesthesia and neuraxial blocks have been successfully used for elective or emergency Caesarean section. Combined spinal epidural anaesthesia (CSE) is preferred by some. CSE causes less haemodynamic instability, has a higher success rate than epidural anaesthesia, results in better patient satisfaction, and provides good postoperative analgesia.

\section{Takotsubo cardiomyopathy}

Recently, Takotsubo cardiomyopathy has been described. Takotsubo cardiomyopathy is a rare condition of transient, reversible severe LV dysfunction and characterised by chest pain, dyspnoea, ST-T changes in ECG, ventricular arrhythmias, regional wall motion abnormalities on echocardiography, elevated cardiac enzymes, haemodynamic instability, pulmonary oedema, cardiogenic shock, or cardiac arrest without angiographic evidence of CAD.

\subsection{Sign and symptoms}

It is rare, usually occurs in postmenopausal women associated with stress and chest pain. ECG changes may include prolonged QTc interval which resolve in 1-2 days, ST-T changes, Q waves, resolve by discharge from hospital, and $\mathrm{T}$ inversion resolves slowly.

This condition is also known as apical ballooning syndrome and broken heart syndrome or stress-induced cardiomyopathy. Echocardiography shows akinesia of apical or midventricular segments leading to systolic dysfunction. The normal basal segments become hypercontractile, giving a ballooned-out appearance of the apical or mid-cavity segments. Ballooning may lead to altered spatial relationships between mitral leaflets and subvalvular apparatus, which may result in MR and dynamic LVOTO causing SAM.

Reversible myocardial ischaemia is seen on myocardial perfusion imaging, and positron emission tomography and magnetic resonance imaging confirm LV dysfunction. Biopsy may show lymphocytic infiltrates. Plasma levels of brain natriuretic peptide, catecholamines, cardiac enzymes and metanephrine are found to be elevated. 


\subsection{Management}

Optimal therapy is yet to be defined [9]. Beta blockers, diuretics, and ACE inhibitor and vasodilators have been used. Adrenergic agonists and antiadrenergic therapy (beta adrenergic blockers or alpha 2 agonists) and QT prolonging medications are to be avoided.

\subsection{Anaesthetic management}

A principle anaesthetic goal is to avoid psychological and physical stress that could trigger acute cardiomyopathy in susceptible patients. Thorough patient counselling, effective premedication and preoperative beta blocker therapy before transfer to operating room are highly effective.

Laryngoscopy, intubation, extubation, emergence, and inadequate postoperative pain control may cause a sympathetic response and increase catecholamine levels, so an optimal anaesthesia/analgesia is required in these phases. It is suggested that regional anaesthesia may be beneficial, but adequate studies to support this theory are not available.

It is unclear whether administration of inotropic drugs to treat systolic dysfunction is harmful. Inotrope of choice remains unclear, though Milrinone, aphosphodiesterase inhibitor, and a calcium sensitizer, levosimendan are suggested. Mechanical support of circulation with IABP or LVAD is an option to tide over periods of crisis. Beta blocker therapy may not be haemodynamically tolerated or could be potentially hazardous. Beta agonists should be avoided or used carefully, vasopressors may be used and supportive treatment for CHF should be instituted. LV dysfunction resolves within 2-4 weeks. Most cases recover spontaneously with a mortality risk of $0-8 \%$.Recurrence occurs in $2-5 \%$ cases [10].

\section{Intensive care unit management}

When a person is admitted with a diagnosis of cardiomyopathy, the main aims of therapy whether in the intensive care or coronary care unit are, to reduce the workload of the heart and to improve the pumping ability of the heart. This can be achieved with the help of drugs such as inotropes, diuretics, ACE inhibitors, beta blockers, calcium channel blockers, and so on, which aids in improving the pumping action of the heart muscle and treatment to ensure the proper volume of blood in the body.

Treatment of the patients in the intensive care unit depends not only on the type and the severity of cardiomyopathy but also condition of patient. Treatment may include conservative management with drugs, implantation of pacemakers, defibrillators for those prone to fatal heart rhythms, ventricular assist devices or extracorporeal membrane oxygenators for severe heart failure, or ablation for recurring dysrhythmias that cannot be managed by drugs or cardioversion. The goal of management in the intensive care unit is often symptomatic, and some patients may eventually require a heart transplant. 


\section{Conclusion}

Nowadays, cardiomyopathies are being identified increasingly as a result of improved means of detection with echocardiographic examination and an increase in the ageing population group. In addition, the presentation of this disease is varied. It may be sudden or already well known to the patient. Anaesthesia administration for patients with cardiomyopathy can lead to perioperative morbidity and mortality during elective or, more importantly in emergency surgeries. Therefore, anaesthesia and postoperative care have to be carefully titrated, planned, and monitored for every patient, for which we need a thorough understanding of the pathophysiology of cardiomyopathies. The best approach would be a multidisciplinary team that includes anaesthetists, cardiologists, and surgeons. As anaesthesiologists, we have to expand our horizon from operating room to ICU with a thorough understanding of non-invasive and invasive monitoring methods and a basic knowledge of transthoracic echocardiography.

\section{Abbreviations}

AHA

ARSA

ARVC

AS

CAD

CHF

CMR

$\mathrm{CO}$

CTR-D

CVP

DCM

EF

EMB

HCM

IABP

ICD
American Heart Association

American Society of Regional Anaesthesia

arrhythmogenic right ventricular cardiomyopathy

aortic stenosis

coronary artery disease

congestive heart failure

cardiac magnetic resonance

cardiac output

cardiac resynchronisation therapy device

central venous pressure

dilated cardiomyopathy

ejection fraction

endomyocardial biopsy

hypertrophic cardiomyopathy

intra-arterial blood pressure

implantable cardioverter-defibrillator 
LA left atrium

LV left ventricle

LVAD left ventricular assist device.

LVEDP left ventricular end-diastolic pressure

LVEF left ventricular ejection fraction

LVOTO left ventricular outflow tract obstruction

NYHA New York Heart Association

PPCM peripartum cardiomyopathy

PPV pulse plethysmographic variability

PVC premature ventricular complex

RCM restrictive cardiomyopathy

SAM systolic anterior moment

$\mathrm{ScVO}_{2} \quad$ central venous oxygen saturation

SVR systemic vascular resistance

TEE trans esophageal echocardiography

VT ventricular tachycardia

WHO World Health Organisation

\section{Author details}

Nandita Mehta* and Sayyidah Aasima tu Nisa Qazi

*Address all correspondence to: drnanditamehta@gmail.com

Department of Anaesthesia and Critical Care, Acharya Shri Chander College of Medical Sciences and Hospital, Jammu, India

\section{References}

[1] Maron BJ, Towbin JA, Thiene G, Antzelevitch C, Corrado D, Arnett D, et al. Contemporary definitions and classification of the cardiomyopathies: An American Heart Association Scientific Statement from the Council on Clinical Cardiology, Heart Failure and Transplantation Committee; Quality of Care and Outcomes Research and Functional Genomics and Translational Biology Interdisciplinary Working Groups; and Council on Epidemiology and Prevention. Circulation. 2006;113:1807-1816 
[2] Elliott P, Andersson B, Arbustini E, Bilinska Z, Cecchi F, Charron P, et al. Classification of the cardiomyopathies: A position statement from the European Society of Cardiology Working Group on myocardial and pericardial diseases. European Heart Journal. 2008;29:270-276

[3] Arbustini E, Narula N, Dec GW, Reddy KS, Greenberg B, Kushwaha S, et al. The MOGE(S) classification for aphenotype-genotype nomenclature of cardiomyopathy: Endorsed by the World Heart Federation. Journal of the American College of Cardiology. 2013;62:2046-2072

[4] Stoelting RK, Dierdorf SF. Cardiomyopathy. In: Stoelting RK, editor. Anaesthesia and Coexisting Disease. 3rd ed. New York: Churchill Livingstone; 1993. pp. 97-102

[5] Abozguia K, Elliott P, McKenna W, Phan TT, Nallur-Shivu G, Ahmed I, Maher AR, Kaur K, Taylor J, Henning A, Ashrafian H, Watkins H, Frenneaux M. Metabolic modulator perhexiline corrects energy deficiency and improves exercise capacity in symptomatic hypertrophic cardiomyopathy. Circulation. 2010;122:1562-1569

[6] Ammash NM, Seward JB, Bailey KR, et al. Clinical profile and outcome of idiopathic restrictive cardiomyopathy. Circulation. 2000;101:2490-2496

[7] Basso C, Corrado D, Bauce B, et al. Arrhythmogenic right ventricle cardiomyopathy. Circulation. Arrhythmia and Electrophysiology. 2012;5:1233-1246

[8] Shnaider R, Ezri T, Szmuk P, et al. Combined spinalepidural anesthesia for cesarean section in a patient with peripartum dilated cardiomyopathy. Canadian Journal of Anesthesia. 2001;48:681-683

[9] Banerjee S. Takotsubo cardiomyopathy: A review. JSM Atherosclerosis. 2016;1:1011

[10] Littlejohn FC, Syed O, Ornstein E, Connolly ES, Heyer EJ. Takotsubo cardiomyopathy associated with anesthesia: Three case reports. Cases Journal. 2008;1:227 
